\title{
Spin freezing by Anderson localization in one-dimensional semiconductors
}

\author{
C. Echeverría-Arrondo \\ Department of Physical Chemistry, Universidad del Pais Vasco UPV/EHU, 48080 Bilbao, Spain \\ E. Ya. Sherman \\ Department of Physical Chemistry, Universidad del País Vasco UPV/EHU, 48080 Bilbao, Spain and \\ IKERBASQUE Basque Foundation for Science, 48011 Bibao, Bizkaia, Spain
}

(Dated: July 13, 2018)

\begin{abstract}
One-dimensional quantum wires are considered as prospective elements for spin transport and manipulation in spintronics. We study spin dynamics in semiconductor GaAs-like nanowires with disorder and spin-orbit interaction by using a rotation in the spin subspace gauging away the spinorbit field. At a strong enough disorder spin density, after a relatively fast relaxation stage, reaches a plateau, which remains a constant for long time. This effect is a manifestation of the Anderson localization and depends in a universal way on the disorder and the spin-orbit coupling strength. As a result, at a given disorder, semiconductor nanowires can permit a long-term spin polarization tunable with the spin-orbit interactions.
\end{abstract}

PACS numbers: 72.25.Rb,72.70.+m,78.47.-p

\section{INTRODUCTION}

The main idea of spintronics - the design and application of devices controlling not only the charge dynamics but also the electron spin evolution - can be useful for information storage, transfer, and manipulation technologies $\underline{\underline{1}-\underline{3}}$ Possible realizations of spintronics devices can be based on semiconductor nanowires $\underline{\underline{4}-10}$ for quasi-ballistic electron transport, coherent transmission of information, and spin control. These systems attract a great deal of attention due to a clear interplay of transport and spin-orbit (SO) coupling physics $\underline{11}-16$

This control faces the problem of inevitable spin relaxation due to the coupling of electron spin to environment through SO coupling. As a result, the factors determining the spin relaxation rate become of crucial importance. Two limiting cases of spin relaxation are well understood. For the itinerant electrons spin relaxation in mainly determined by the Dyakonov-Perel' mechanism, that is by random precession of electron spin due to the random in time electron momentum.

A different approach should be applied for electrons localized in a regular external potential forming quantum dots promising for quantum information applications $\stackrel{17}{\underline{17}}$ Here momentum is not a well-defined quantity, and the momentum-dependent splitting required for the Dyakonov-Perel mechanism vanishes. As a result, spin relaxation through $\mathrm{SO}$ interaction requires phonon-induced coupling of different orbital states of the localized electron and nonzero external magnetic field $\underline{\underline{18}}$ In the absence of magnetic field and spin-orbit coupling, spin relaxation can occur due to the hyperfine coupling of electron spin to spins of lattice nuclei $\frac{19}{19}$ In both cases, the initial spin polarization goes asymptotically to zero. The characteristic timescale of spin relaxation of electrons localized in quantum dots is expected to be several orders of magnitude longer than that of itinerant electrons.

While these two limits of free and strongly localized

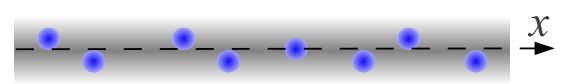

FIG. 1: (Color online) Semiconductor nanowire with random impurities shown as filled circles. Although we consider a one-dimensional electron motion, impurities can be randomly distributed over the cross-section of the wire.

electrons are well understood, the interplay of disorderinduced localization and spin relaxation of itinerant electrons remains an open question although some aspects of the problem have been addressed $\underline{20} \underline{23}$ The questions here are (i) how the localization forms the spin relaxation, and (ii) whether the initially prepared spin density relaxes to zero. As a nontrivial example of this interplay we mention that weak localization of two-dimensional electrons leads to a long power-like rather than exponential spin relaxation. ${ }^{24,25}$ Here we analyze this problem for the one-dimensional system, providing, on one hand, the basic example of localization physics in a random potential, $, 26,27$ and, on the other hand, an example of a system, where spin-orbit coupling can be gauged away by a $\mathrm{SU}(2)$ transformation.

This paper is organized as follows. In Sec. II, we show how to treat spin relaxation in one-dimensional systems with the gauge transformation and introduce the tightbinding Hamiltonian for the model. The spin dynamics will be analyzed by a numerically exact calculation in Sec. III, where we show that spin density does not relax to zero, in contrast to what expected. In addition, in this Sec. III we study how asymptotic value of spin polarization depends on the disorder and spin-orbit coupling. Conclusions summarize the results in Sec. IV. 


\section{MODEL}

\section{A. Hamiltonian and gauge transformation}

The investigated structure is a quantum wire extended along the $x$ axis, as shown in Fig. 11. The total Hamiltonian has the form

$$
\hat{H}=\frac{\hbar^{2}}{2 m}\left(k_{x}-A_{x}\right)^{2}+U(x)-\frac{m \alpha^{2}}{2 \hbar^{2}},
$$

where $A_{x}=-m \alpha \sigma_{y} / \hbar^{2}$ stands for the Rashba coupling 28 with the strength $\alpha, \sigma_{y}$ is the Pauli matrix, $k_{x}$ is the electron wavevector, and $m$ is the effective mass. The Dresselhaus coupling 29 is obtained with $A_{x}=-m \beta \sigma_{x} / \hbar^{2}$, where $\beta$ is the coupling constant. Without loss of generality, we concentrate here on the Rashba coupling, which can be changed on demand by applying external electric field across the structure $\underline{30}$

The SO interaction can be removed from $\hat{H}$ in Eq. (1) through a gauge transformation ${ }^{31.32}$ with a $\mathrm{SU}(2)$ spin rotation: $\hat{S}=\exp \left(-i x \sigma_{y} / 2 \xi\right)$, where $\xi=\hbar^{2} / 2 m \alpha$ is the spin-precession length. After this transformation the system Hamiltonian has the form: $\hat{\widetilde{H}}=\hbar^{2} k_{x}^{2} / 2 m+U(x)$.

Since for the Hamiltonian (11), $\sigma_{y}$ is the integral of motion, the spin density component along the $y$-axis is time independent. A nontrivial dynamics of the transformed spin occurs for the $\gamma=(x, z)$ spin components $\left\langle\widetilde{s}_{\gamma}(x, t)\right\rangle$ and can be expressed in terms of the spin diffusion

$$
\left\langle\widetilde{s}_{\gamma}(x, t)\right\rangle=\int D^{\gamma \beta}\left(x-x^{\prime}, t\right)\left\langle\widetilde{s}_{\beta}\left(x^{\prime}, 0\right)\right\rangle d x^{\prime},
$$

where $D^{\gamma \beta}(x, t)$ is the exact disorder-dependent onedimensional spin diffusion Green's function. In a nonmagnetic system without SO coupling $D^{\gamma \beta}(x, t)=$ $\delta_{\gamma \beta} D(x, t)$ is diagonal in the spin subspace. As a result of the gauge transformation, the uniform density dynamics is determined by only the Fourier component 25

$$
D(q, t)=\int_{-\infty}^{\infty} d x e^{-i q x} D(x, t)
$$

with $q=1 / 2 \xi$ and Eq. (2) simplifies for the physical measurable spins as $\left\langle s_{\gamma}(t)\right\rangle=\left\langle s_{\gamma}(0)\right\rangle D(1 / 2 \xi, t)$. Here we will use a similar, however, somewhat different approach based on numerically exact analysis of the direct time evolution of the initial spin-polarized states. It will be shown that the resulting spin dynamics has unexpected features, including a long-time plateau in the spin polarization.

The eigenfunctions of $\hat{\widetilde{H}}$ can be taken in the form $\bar{\psi}(x)=\psi(x)|1\rangle$ and $\bar{\psi}(x)=\psi(x)|-1\rangle$, where $| \pm 1\rangle$ are the eigenstates of $\sigma_{z}$ with the corresponding eigenvalues. The eigenstates of $\hat{H}, \bar{\phi}(x)$ can be obtained by spin rotation of the $\bar{\psi}(x)|\sigma\rangle$ states. For example, with spin-up initial state $\bar{\psi}(x)|1\rangle$ one obtains:

$$
\bar{\phi}(x)=\psi(x)\left[\cos \left(\frac{x}{2 \xi}\right)|1\rangle+\sin \left(\frac{x}{2 \xi}\right)|-1\rangle\right] .
$$

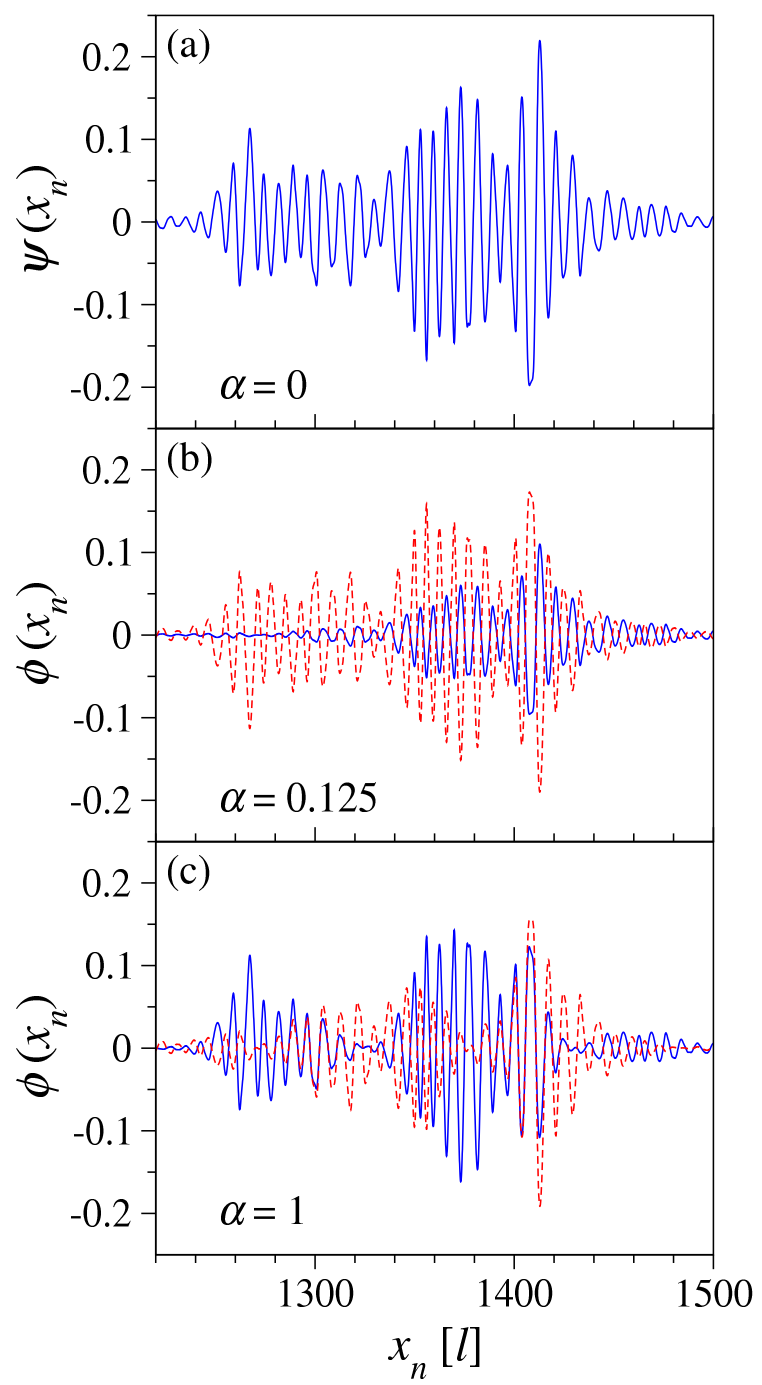

FIG. 2: (Color online) Site dependent components of $\bar{\phi}_{N / 4}\left(x_{n}\right)$ for a qualitative description of entanglement induced by the gauge transformation for (a) $\alpha=0$, (b) $\alpha=$ $0.125 \times 10^{-6} \mathrm{meVcm}$, and (c) $\alpha=10^{-6} \mathrm{meVcm}\left(U_{0}=55\right.$ $\mathrm{meV})$. The solid and dashed lines represent $|1\rangle$ and $|-1\rangle$ components, respectively.

The spin dynamics and spin relaxation in the system, as it will be shown below, is solely due to the entanglement of spin and coordinate in Eq.(4).

\section{B. Tight-binding model and disorder}

We perform numerical analysis using the tight-binding model, employing the approach similar to Refs. [16, 33]. The one-dimensional electron gas is sampled with $N=$ $2^{13}$ (8192) grid points $x_{n}=n l$, where $1 \leq n \leq N$ and $l$ is the effective lattice constant with periodic boundary conditions .34 The effective hopping matrix element between two nearest neighbors is chosen as $t=50 \mathrm{meV}$, and the kinetic energy is $E\left(k_{x}\right)=2 t\left(1-\cos \left(k_{x} l\right)\right)$. As a 
result, the eigenenergies span the range of $[0,200] \mathrm{meVs}$. The distance between two neighbor grid points becomes $l=\hbar / \sqrt{2 m t}=3.37 \mathrm{~nm}$ to satisfy the electron effective mass $m=0.067 m_{0}$ in GaAs semiconductor with $m_{0}$ being the free electron mass.

The random potential $U_{n}=U\left(x_{n}\right)$ uniformly spans the range $\left[-U_{0} / 2, U_{0} / 2\right]$ with the white noise correlator $\left\langle U\left(x_{n_{1}}\right) U\left(x_{n_{2}}\right)\right\rangle=\left\langle U^{2}\right\rangle \delta_{n_{1}, n_{2}}$, where $\left\langle U^{2}\right\rangle=U_{0}^{2} / 12$. The effects of disorder can be approximately characterized through the energy-dependent momentum relaxation time $\tau_{E}$, which we define as $\hbar / \tau_{E}=\left\langle U^{2}\right\rangle l \nu_{E}$, where $\nu_{E}=\sqrt{m} / \pi \hbar \sqrt{2 E}$ is the density of states per spin component. The resulting mean free path $\ell_{E}=v_{E} \tau_{E}$, where $v_{E}=\sqrt{2 E / m}$ is the electron speed and the corresponding diffusion coefficient $D_{E}=v_{E}^{2} \tau_{E}$.

In this representation the eigenstates of $\hat{\widetilde{H}}$ and $\hat{H}$ form basis sets, $\left\{\bar{\psi}_{i}\right\}$ and $\left\{\bar{\phi}_{i}\right\}$ respectively, where $1 \leq i \leq 2 N$. For the same $i$, these two sets are related by the local spin rotation $\hat{S}$. We assume that $\bar{\psi}_{i}=\psi_{i}\left(x_{n}\right)|1\rangle$ for $1 \leq i \leq N$ and $\bar{\psi}_{i}=\psi_{i-N}\left(x_{n}\right)|-1\rangle$ for $N<i \leq 2 N$.

\section{SPIN DYNAMICS}

We study dynamics of initial $\bar{\psi}_{i}$ states with $1 \leq i \leq N$, corresponding to the evolution upon instant switching of the SO coupling. The time dependence can be expressed with the spectral decomposition as:

$$
\bar{\psi}_{j}^{\mathrm{so}}(t)=\sum_{i=1,2 N} a_{i j} \bar{\phi}_{i} e^{-i t \varepsilon_{i} / \hbar},
$$

where $a_{i j}=\left\langle\bar{\phi}_{i} \mid \bar{\psi}_{j}\right\rangle$, and $\varepsilon_{i}$ are the corresponding eigenenergies. The spin component expectation value $\left\langle\sigma_{z}(t)\right\rangle_{j}=\left\langle\bar{\psi}_{j}^{\mathrm{so}}(t)\left|\sigma_{z}\right| \bar{\psi}_{j}^{\mathrm{so}}(t)\right\rangle$ is determined by the spectrum and eigenstates of the system.

In order to give an idea of the entanglement induced by $\mathrm{SO}$ coupling, we present in Fig. 2 the evolution of $\bar{\phi}_{N / 4}\left(x_{n}\right)$ state with the increase in the spin-orbit coupling. At $\alpha=0$, we obtain a product state $\bar{\phi}_{N / 4}\left(x_{n}\right)=$ $\psi_{N / 4}\left(x_{n}\right)|1\rangle$, and with the increase in $\alpha$ entangled states are formed. The overlap of $\bar{\phi}_{i}\left(x_{n}\right)$ and $\bar{\psi}_{j}\left(x_{n}\right)$ eigenstates is characterized by two sets of matrix elements $a_{i j}$; for example, for $1 \leq j \leq N$ :

$$
\begin{array}{ll}
a_{i j}=\sum_{n} \cos \left(\frac{x_{n}}{2 \xi}\right) \psi_{i}\left(x_{n}\right) \psi_{j}\left(x_{n}\right), \quad 1 \leq i \leq N \\
a_{i j}=\sum_{n} \sin \left(\frac{x_{n}}{2 \xi}\right) \psi_{i}\left(x_{n}\right) \psi_{j}\left(x_{n}\right), \quad N<i \leq 2 N .
\end{array}
$$

The behavior of $a_{i j}$ presented Fig. 3 demonstrates that for given $j$ it has nonnegligible values only in a certain, rather narrow, range of $i$.

To illustrate the role of the random potential, we consider as examples weak $\left(U_{0}=15 \mathrm{meV}, U_{0} \ll t\right)$ and

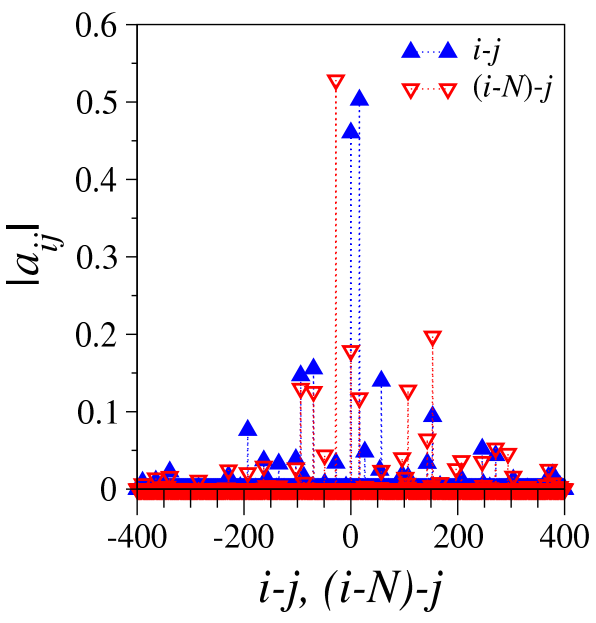

FIG. 3: (Color online) Absolute values of $a_{i j}$ around the initial spin-up state $\bar{\psi}_{N / 4}$; here $\alpha=10^{-6} \mathrm{meVcm}$ (strong SO coupling) and $U_{0}=55 \mathrm{meV}$ (strong disorder).

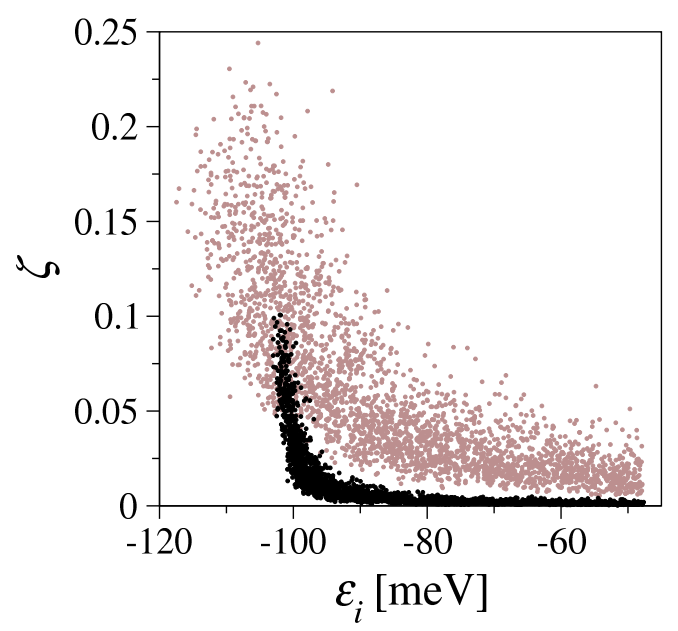

FIG. 4: (Color online) Inverse participation ratio $\zeta$ for the low part of the energy spectrum; gray (red) circles denote strong disorder $\left(U_{0}=55 \mathrm{meV}\right)$ and black circles denote weak disorder $\left(U_{0}=15 \mathrm{meV}\right)$. Since even for $U_{0}=55 \mathrm{meV}$ we obtain $\zeta \ll 1$, the localized states are distributed over many lattice sites, confirming applicability of the tight-binding Hamiltonian for the localization problem.

strong $\left(U_{0}=55 \mathrm{meV}, U_{0}>t\right)$ disorder. For free electrons in state $j=N / 4$ and $E=31 \mathrm{meV}$, the resulting $\hbar / \tau_{E}$ is about 0.1 and $1 \mathrm{meV}$, respectively. For a free electron with the energy $E \approx 20 \mathrm{meV}$ the velocity $v_{E} \approx 3.5 \times 10^{7} \mathrm{~cm} / \mathrm{s}$, the mean free path $\ell_{E} \sim 2.5 \times 10^{-5}$ $\mathrm{cm}\left(\hbar / \tau_{E}=1 \mathrm{meV}\right)$, and the corresponding diffusion coefficient $D_{E} \sim 10^{3} \mathrm{~cm}^{2} / \mathrm{s}$. These parameters provide an effective integral characteristic of the disorder and correspond to realistic parameters of the wires, which, however, can strongly vary from sample to sample and from experiment to experiment.

The effect of localization by disorder is seen in the inverse participation ratio $\underline{35}$ (IPR) $\zeta_{i}=\sum_{n}\left|\psi_{i}^{4}\left(x_{n}\right)\right|$. The 


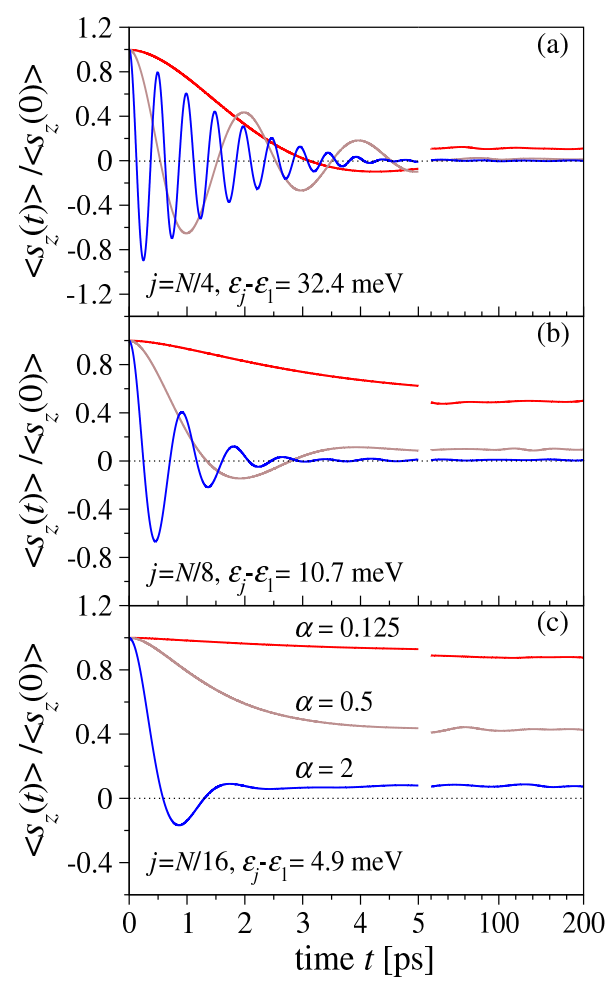

FIG. 5: (Color online) Time-dependent polarization in the weak-disorder regime $\left(U_{0}=15 \mathrm{meV}\right)$. The initial bins are centered at the states (a) $N / 4$ (bin width $6.8 \mathrm{meV}$ ), (b) $N / 8$ (bin width $3.7 \mathrm{meV}$ ), and (c) and $N / 16$ (bin width $2.1 \mathrm{meV}$ ) with energies decreasing in the same order. The curves for SO couplings $0.125 \times 10^{-6} \mathrm{meVcm}, 0.5 \times 10^{-6} \mathrm{meVcm}$, and $2 \times 10^{-6} \mathrm{meVcm}$ are drawn with circles, triangles, and squares, respectively. Note that after the relaxation stage the spin density remains a finite constant.

IPR calculated for the low-energy spectrum is presented in Fig. 4. As expected, the degree of localization increases with $U_{0}$ and this effect is more pronounced for the electrons with lowest energies. In contrast to the results of Ref. 23], the IPR in this system does not depend on the SO coupling. We now study the effects of disorder and spin-orbit coupling on the average spin dynamics of a bin of 256 initial spin-up states and 8 realizations of the random potential. The statistical error of this approach is, therefore $1 / \sqrt{2048}=2.2 \%$, making the results statistically representative.

We take three example bins with three different degrees of localization. The bins are centered around the spin-up states $\bar{\psi}_{N / 4}, \bar{\psi}_{N / 8}$, and $\bar{\psi}_{N / 16}$, whose IPR values increase in the same order (energies decreasing, see Fig. (4). The calculated bin- and potential realizationaveraged spin dynamics is shown in Figs. 5 and 6 revealing strong influence of the disorder-induced spatial localization of states. Physically, collisions of electrons with impurities force electron spin to frequently reverse the precession direction. In the classical picture, this leads to a long Dyakonov-Perel' spin relaxation. If the

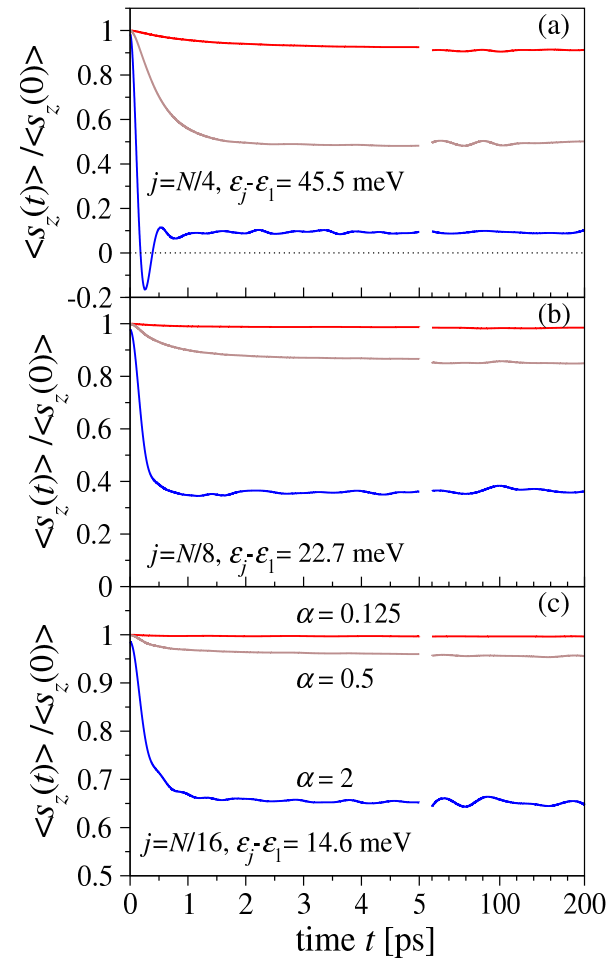

FIG. 6: (Color online) Time-dependent spin polarization in the strong-disorder regime $\left(U_{0}=55 \mathrm{meV}\right)$ with the same notations as in Fig. 5] (a) $N / 4$ (bin width $7.2 \mathrm{meV}$ ), (b) $N / 8$ (bin width $4.5 \mathrm{meV}$ ), and (c) $N / 16$ (bin width $3.7 \mathrm{meV}$ ). Note that for $\alpha=0.125 \times 10^{-6} \mathrm{meVcm}$ the spin is almost constant in time, thus suitable for spin-based operations.

quantum effects of localization are important, the resulting effect is the "freezing" of the electronic spin. As one can see in Figs. 5 and 6, the electron spin density relaxes for $\simeq 5 \mathrm{ps}$ and then remains constant in time for infinitely long (beyond $0.2 \mathrm{~ns}$ in our computation). As expected, the spin polarization plateau is higher (i) for localized states and (ii) for weak SO interaction. Almost time-independent spin states are achieved e.g., at $U_{0}=55 \mathrm{meV}$ and $\alpha=0.125 \times 10^{-6} \mathrm{meVcm}$.

To gain insight into the problem, we study the dependence of asymptotic spin density on SO coupling and the localization of electrons in more detail. The long-term densities are plotted in Fig. 7 against parameter $\xi\langle\zeta\rangle$. This parameter combines the two factors determining the spin dynamics, SO coupling and spatial localization, where $\langle\zeta\rangle$ is averaged over 256 bin states and 8 realization of the random potential. The given values follow a universal dependence indicating a unique trend for longterm spins against SO coupling and localization through disorder. This trend corresponds to a fast increase in the asymptotic steady polarization for $\xi\langle\zeta\rangle<1$ and a smooth increase and saturation for $\xi\langle\zeta\rangle>1$. These results can be understood as follows. To show an efficient spin dynamics, the electron should move the distance of the order of $\pi \xi$. Therefore, the spatial spread of the correspond- 


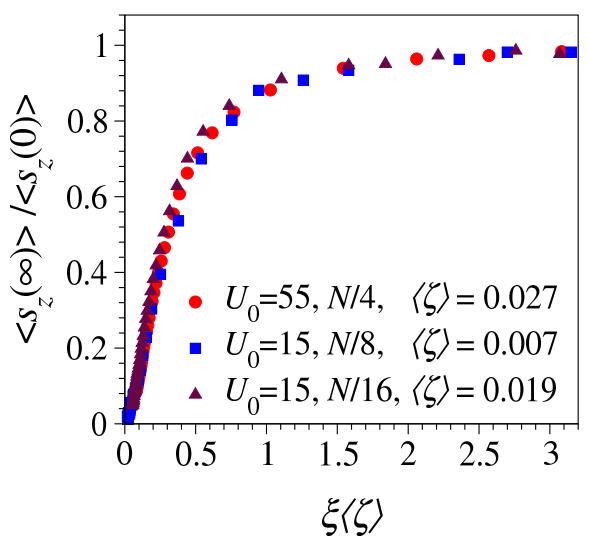

FIG. 7: (Color online) Long-term relative polarization as a function of $\xi\langle\zeta\rangle$ for three different degrees of localization. Parameter $\xi$ is modified by changing the coupling constant $\alpha$.

ing states should be larger than $\pi \xi$. With a stronger localization, the spread and the overlap decrease leading to the universal behavior shown in Fig:7. Qualitatively, in the "clean" $\xi\langle\zeta\rangle \ll 1$ regime the spin relaxation has the Dyakonov-Perel' mechanism either purely exponential for $\Omega_{E} \tau_{E} \ll 1$ or a combination of oscillations and exponential decay if $\Omega_{E} \tau_{E} \geq 1$, where the spin precession rate $\Omega_{E}=2 \alpha \sqrt{2 m E} / \hbar$ corresponds to the electron momentum at given energy $E$.

\section{CONCLUSION}

To summarize, localization effects of disorder and SO coupling in semiconductor nanowires determine the dynamics of electronic spins. Our tight-binding model cal- culations show that a prepared spin density relaxes until reaching a plateau, directly related to the disorder and strength of SO interaction. In contrast to the expected decay to zero, a long-time constant polarization plateau survives to infinite time. The asymptotic spin density has a universal dependence on the product of the inverse participation ratio and the spin precession length. In the absence of magnetic field, the hyperfine coupling to the spins of nuclei will lead to spin relaxation on timescales at least two orders of magnitude longer than the timescale of the plateau formation of the order of $10 \mathrm{ps}: \frac{19}{1}$ As the experiments on spin transport did not reveal electron-electron interaction effects,,$\frac{8}{,}$ here we have neglected them. Furthermore, whether there exists a range of parameters where the Coulomb forces can be strong enough to modify our results for localized states, remains to be investigated.

An immediate consequence of this result is the ability, by choosing the desired Rashba SO parameter for a given wire, to produce and destroy steady spin states, which are of interest for spin-based operations. These results suggest that semiconductor nanowires can be used for coherent transmission and storage of information, manipulated by spatially and temporally modulated spin-orbit coupling.

\section{ACKNOWLEDGMENTS}

We thank G. Japaridze, J. Siewert, and L.A. Wu for helpful discussions. This work was supported by the MCI of Spain grant FIS2009-12773-C02-01 and "Grupos Consolidados UPV/EHU del Gobierno Vasco" grant IT-47210.
1 I. Zutić, J. Fabian, and S. Das Sarma, Rev. Mod. Phys. 76, 323 (2004); J. Fabian, A. Matos-Abiague, C. Ertler, P. Stano, and I. Zutic, Acta Physica Slovaca 57, 565 (2007).

2 A. Fert, Rev. Mod. Phys. 80, 1517 (2008).

${ }^{3}$ M. W. Wu, J. H. Jiang, and M. Q. Weng, Physics Reports 493, $61(2010)$.

4 X. Duan, Y. Huang, Y. Cui, J. Wang, and C. M. Lieber, Nature 409, 66 (2001).

5 S. Nadj-Perge, S. Frolov, E. Bakkers, and L. Kouwenhoven, Nature 468, 1084 (2010).

6 S. Pramanik, S. Bandyopadhyay, and M. Cahay, Phys. Rev. B 68, 075313 (2003).

7 A. A. Kiselev and K. W. Kim, Phys. Rev. B 61, 13115 (2000).

8 C. H. L. Quay, T. L. Hughes, J. A. Sulpizio, L. N. Pfeiffer, K. W. Baldwin, K. W. West, D. Goldhaber-Gordon, and R. de Picciotto, Nat. Phys 6, 336 (2010).

9 A. Bringer and T. Schäpers, Phys. Rev. B 83, 115305 (2011).

10 M. Governale and U. Zülicke, Phys. Rev. B 66, 073311 (2002).
11 Y. V. Pershin, J. A. Nesteroff, and V. Privman, Phys. Rev. B 69, 121306 (2004); V. A. Slipko, I. Savran, and Y. V. Pershin, Phys. Rev. B 83, 193302 (2011).

12 A. W. Holleitner, V. Sih, R. C. Myers, A. C. Gossard, and D. D. Awschalom, Phys. Rev. Lett. 97, 036805 (2006).

13 M. M. Gelabert, L. Serra, D. Sánchez, and R. López Phys. Rev. B 81, 165317 (2010); D. Sánchez, L. Serra, and M.-S. Choi Phys. Rev. B 77, 035315 (2008).

14 C. Lü, U. Zülicke, and M. W. Wu, Phys. Rev. B 78, 165321 (2008)

15 C. L. Romano, P. I. Tamborenea, and S. E. Ulloa, Phys. Rev. B 74, 155433 (2006)

16 G. I. Japaridze, H. Johannesson, and A. Ferraz, Phys. Rev. B 80, 041308 (2009); M. Malard, I. Grusha, G. I. Japaridze, and H. Johannesson, Phys. Rev. B 84, 075466 (2011).

17 G. Burkard, D. Loss, and D. P. DiVincenzo, Phys. Rev. B 59, 2070 (1999); L.-A. Wu and D. A. Lidar, Phys. Rev. Lett. 91, 097904 (2003); L.-A. Wu, D. A. Lidar and M. Friesen, Phys. Rev. Lett. 93, 030501 (2004).

18 P. Stano and J. Fabian, Phys. Rev. Lett. 96, 186602 (2006); 
P. Stano and J. Fabian, Phys. Rev. B 74, 045320 (2006).

19 I. A. Merkulov, Al. L. Efros, and M. Rosen, Phys. Rev. B 65, 205309 (2002).

20 T. P. Pareek and P. Bruno, Phys. Rev. B 65, 241305 (2002).

21 B. I. Shklovskii, Phys. Rev. B 73, 193201 (2006).

22 T. Kaneko, M. Koshino, and T. Ando, Physica E 40, 383 (2007).

${ }^{23}$ G. A. Intronati, P. I. Tamborenea, D. Weinmann, and R. A. Jalabert, arXiv:1102.4753.

24 I. S. Lyubinskiy and V. Yu. Kachorovskii, Phys. Rev. B 70, 205335 (2004).

25 I. V. Tokatly and E. Ya. Sherman, Phys. Rev. B 82, 161305 (2010).

${ }^{26}$ P. W. Anderson, D. J. Thouless, E. Abrahams, and D. S. Fisher, Phys. Rev. B 22, 3519 (1980).

27 I. M. Lifshitz, S. A. Gredeskul, and L. A. Pastur, Introduction to the Theory of Disordered Systems, Wiley, NY, (1988); A. L. Efros and B. I. Shklovskii, Electronic Properties of Doped Semiconductors, Springer, Heidelberg, (1989).

28 Yu. A. Bychkov and E. I. Rashba, JETP Lett. 39, 79 (1984).

29 G. Dresselhaus, Phys. Rev. 100, 580 (1955).

30 D. Grundler, Phys. Rev. Lett. 84, 6074 (2000); O. Z. Karimov, G. H. John, R. T. Harley, W. H. Lau, M. E. Flatte,
M. Henini, and R. Airey, Phys. Rev. Lett. 91, 246601 (2003), A. Balocchi, Q. H. Duong, P. Renucci, B. L. Liu, C. Fontaine, T. Amand, D. Lagarde, and X. Marie, Phys. Rev. Lett. 107, 136604 (2011).

31 L. S. Levitov and E. I. Rashba, Phys. Rev. B 67, 115324 (2003).

32 I. Tokatly and E. Ya. Sherman, Annals of Phys. 325, 1104 (2010).

33 E. M. Hankiewicz, L. W. Molenkamp, T. Jungwirth, and J. Sinova, Phys. Rev. B 70, 241301 (2004).

34 Since we are studying the spin dynamics in the lower part of the spectrum, the matrix Hamiltonian is only partially diagonalized up to 2800 eigenenergies and eigenstates. We use for this purpose the Lanczos method as implemented in the PARPACK package, a collection of Fortran routines for parallel computing. See www.caam.rice.edu/software/ARPACK/index.html for more information about the parallel version of ARPACK.

35 F. Evers and A. D. Mirlin, Phys. Rev. Lett. 84, 3690 (2000).

36 V. N. Gridnev, Pis'ma Zh. Eksp. Teor. Fiz. 74, 417 (2001) [JETP Lett. 74, 380 (2001)]; C. Grimaldi, Phys. Rev. B 72, 075307 (2005); M. M. Glazov, Solid State Commun. 142, 531 (2007); X. Liu, X.-J. Liu, and J. Sinova, Phys. Rev. B 84, 035318 (2011). 